International Journal of Pure and Applied Mathematics

Volume 96 No. 1 2014, 117-134

ISSN: 1311-8080 (printed version); ISSN: 1314-3395 (on-line version)

url: http://www.ijpam.eu

doi: http://dx.doi.org/10.12732/ijpam.v96i1.9

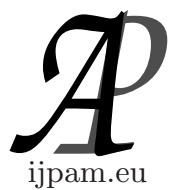

\title{
GLOBAL PROPERTIES OF SOLUTIONS OF FOURTH ORDER GENERALIZED $\alpha$-DIFFERENCE EQUATIONS
}

\author{
M. Maria Susai Manuel ${ }^{1} \S$, K. Srinivasan ${ }^{2}$, D.S. Dilip ${ }^{3}$, G. Dominic Babu ${ }^{4}$ \\ ${ }^{1}$ Department of Science and Humanities \\ R.M.D. Engineering College \\ Kavaraipettai, 601 206, Tamil Nadu, S. INDIA \\ ${ }^{2}$ Department of Science and Humanities \\ S.K.P. Institute of Technology \\ Tiruvannamalai, Tamil Nadu, S. INDIA \\ ${ }^{3}$ Department of Mathematics \\ St. John's College \\ Anchal, Kollam District, Kerala, INDIA \\ ${ }^{4}$ Department of Mathematics \\ Sacred Heart College \\ Tirupattur, 635 601, Vellore District \\ Tamil Nadu, S. INDIA
}

Abstract: In this paper, the authors discuss various properties of solutions for the generalized $\alpha$-difference equation

$$
\Delta_{\alpha(\ell)}^{4} u(k-2 \ell)=\alpha^{2} p(k) u(k), \quad k \in[2 \ell, \infty),
$$

where the functions $p$ is positive on $[2 \ell, \infty), \alpha>1$ and $\ell$ is a positive real.

AMS Subject Classification: 39A, 12

Key Words: generalized $\alpha$-difference equation, generalized $\alpha$-difference operator, oscillation and nonoscillation

Received: July 9, 2014

(C) 2014 Academic Publications, Ltd.

${ }^{\S}$ Correspondence author url: www.acadpubl.eu 


\section{Introduction}

The basic theory of difference equations is based on the operator $\Delta$ defined as $\Delta u(k)=u(k+1)-u(k), k \in \mathbb{N}=\{0,1,2,3, \cdots\}$. Eventhough many authors ([1], [12]-[14]) have suggested the definition of $\Delta$ as

$$
\Delta u(k)=u(k+\ell)-u(k), k \in \mathbb{R}, \ell \in \mathbb{R}-\{0\},
$$

no significant progress took place on this line. But recently, E. Thandapani, M.M.S. Manuel, G.B.A.Xavier [5] considered the definition of $\Delta$ as given in (2) and developed the theory of difference equations in a different direction. For convenience, the operator $\Delta$ defined by (2) is labelled as $\Delta_{\ell}$ and by defining its inverse $\Delta_{\ell}^{-1}$, many interesting results and applications in number theory (see $[5],[7]-[11])$ were obtained. By extending the study related to the sequences of complex numbers and $\ell$ to be real, some new qualitative properties of the solutions like rotatory, expanding, shrinking, spiral and weblike were obtained for difference equations involving $\Delta_{\ell}$. The results obtained using $\Delta_{\ell}$ can be found in ([5]-[11]). Jerzy Popenda and B.Szmanda ([2],[3]) defined $\Delta$ as

$$
\Delta_{\alpha} u(k)=u(k+1)-\alpha u(k)
$$

and based on this definition they studied the qualitative properties of a particular difference equation and no one else has handled this operator. In this paper, we have generalized the definition of $\Delta_{\alpha}$ given in (3) and defined and denoted it as

$$
\Delta_{\alpha(\ell)} u(k)=u(k+\ell)-\alpha u(k),
$$

where $\alpha>1$ and $\ell \in[0, \infty)$. and by defining its inverse, several interesting results on number theory were obtained.

In this paper, we discuss various properties of solutions of (1).

Throughout this paper we use the following notations;

(i) $\mathbb{N}=\{0,1,2,3, \ldots\}, \mathbb{N}(a)=\{a, a+1, a+2, \ldots\}$,

(ii) $\mathbb{N}_{\ell}(j)=\{j, j+\ell, j+2 \ell, \ldots\}$,

(iii) $[x]$ denotes the integer part of $x$.

\section{Preliminaries}

In this section, we present some basic definitions and results which will be useful for further discussion. 
Definition 2.1. [8] Let $u(k), k \in[0, \infty)$ be a real or complex valued function and $\ell \in(0, \infty)$. Then, the generalized $\alpha$-difference operator $\Delta_{\alpha(\ell)}$ on $u(k)$ is defined as in (3). When $\alpha=1$, the generalized $\alpha$-difference operator $\Delta_{\alpha(\ell)}$ becomes the generalized difference operator $\Delta_{\ell}$. When $\alpha=1$ and $\ell=1$, then $\Delta_{\alpha(\ell)}$ becomes the usual difference operator $\Delta$. Similarly, the generalized $\alpha$-difference operator of the $r^{\text {th }}$ kind is defined as

$$
\Delta_{\alpha(\ell)}^{r} u(k)=\underbrace{\Delta_{\alpha(\ell)}\left(\Delta_{\alpha(\ell)}\left(\ldots\left(\Delta_{\alpha(\ell)} u(k)\right)\right)\right)}_{r \text { times }} .
$$

In particular,

$$
\Delta_{\alpha(\ell)}^{4} u(k)=u(k+4 \ell)-4 \alpha u(k+3 \ell)+6 \alpha^{2} u(k+2 \ell)-4 \alpha^{3} u(k+\ell)+\alpha^{4} u(k) .
$$

Definition 2.2. [5] Let $u(k), k \in[0, \infty)$ be a real or complex valued function and $\ell \in(0, \infty)$. Then, the inverse of $\Delta_{\ell}$ denoted by $\Delta_{\ell}^{-1}$ is defined as follows.

$$
\text { If } \Delta_{\ell} v(k)=u(k) \text {, then } v(k)=\Delta_{\ell}^{-1} u(k)+c_{j},
$$

where $c_{j}$ is a constant for all $k \in \mathbb{N}_{\ell}(j), j=k-\left[\frac{k}{\ell}\right] \ell$. In general, $\Delta_{\ell}^{-n} u(k)=$ $\Delta_{\ell}^{-1}\left(\Delta_{\ell}^{-(n-1)} u(k)\right)$ for $n \in \mathbb{N}(2)$.

Definition 2.3. [9] The inverse of the Generalized $\alpha$-difference operator denoted by $\Delta_{\alpha(\ell)}^{-1}$ on $u(k)$ is defined as follows. If $\Delta_{\alpha(\ell)} v(k)=u(k)$, then

$$
\Delta_{\alpha(\ell)}^{-1} u(k)=v(k)-\alpha^{\left[\frac{k}{\ell}\right]} c_{j} .
$$

where $c_{j}$ is a constant for all $k \in \mathbb{N}_{\ell}(j), j=k-\left[\frac{k}{\ell}\right] \ell$.

Definition 2.4. [6] A solution $u(k)$ of (1) is called recessive, if there exists an $a \in[0, \infty)$ such that for all $k \in[a, \infty)$

$$
u(k)>0, \Delta_{\alpha(\ell)} u(k) \leq 0, \Delta_{\alpha(\ell)}^{2} u(k) \geq 0 \text { and } \Delta_{\alpha(\ell)}^{3} u(k) \leq 0 .
$$

Lemma 2.5. [9] Let $\lim _{k \rightarrow \infty} \frac{\left(v(k)-L_{j}\right)}{\alpha^{k}}=L, u(k)=v(k)-L_{j}$ and

$$
\begin{aligned}
\lim _{k \rightarrow \infty} \Delta_{\alpha(\ell)} u(k)=\lim _{k \rightarrow \infty} \Delta_{\alpha(\ell)}^{2} u(k) & =\lim _{k \rightarrow \infty} \Delta_{\alpha(\ell)}^{3} u(k)=0 . \text { Then } \\
\Delta_{\alpha(\ell)}^{-4} u(k) & =\sum_{r=0}^{\infty}\left(\begin{array}{c}
r+3 \\
3
\end{array}\right) \frac{u(k+r \ell)}{\alpha^{r+4}} .
\end{aligned}
$$


Lemma 2.6. If $u$ is a solution of (1) with $u(a+j)=u(a+j+\ell)=$ $u(a+j+2 \ell)=u(a+j+3 \ell)=0$ for all $j \in[0, \ell)$ for some $a \in[0, \infty)$, then $u(k)=0$ for all $k \in[0, \infty)$.

Proof. The proof follows from (1) and (6).

Definition 2.7. Let $u(k)$ be a solution of $(1)$ and we say that $k \in(\ell, \infty)$ is a $\ell$-generalized zero for $u(k)$ if one of the following holds:

(i) $u(k)=0$;

(ii) $k \in(\ell, \infty)$ and $u(k-\ell) u(k)<0 ; k \in(\ell, \infty)$ and there exists an integer $m$, $1<m \leq\left[\frac{k}{\ell}\right]$ such that

(iii) $(-1)^{m} u(k-m \ell) u(k)>0$ and $u(n)=0$ for all $n \in \mathbb{N}_{\ell}(k-m \ell+\ell, k-\ell)$.

An $\ell$-generalized zero for $u(k)$ is said to be of order 0,1 or $m>1$, according to whether the above condition (i), (ii) or (iii), respectively, holds. In particular, an $\ell$-generalized zero of order 0 will simply be called a zero, and a $\ell$-generalized zero of order one will be called a node.

Thus, a nontrivial solution of (1) can have zeros at no more than three consecutive values of $k$. In section 4 , we shall show that a nontrivial solution (1) cannot have a $\ell$-generalized zero of order $m>3$. However, a solution of (1) can have arbitrarily many consecutive nodes, as it is clear from $u(k)=(-\alpha)^{\left[\frac{k}{\ell}\right]}$, which is the solution of $\Delta_{\alpha(\ell)}^{4} u(k-2 \ell)=16 \alpha^{2} u(k)$.

Definition 2.8. [4] A function $u(k)$ is called convex upward on $I$ if its derivative is decreasing on the interval $I$.

\section{Monotonicity Properties}

Lemma 3.1. If $u(k)$ is a function satisfying $\Delta_{\alpha(\ell)}^{2} u(k) \geq 0$ for all $k \in$ $[K, \infty)$ for some $K$, then

$$
u(k) \geq u(K+j)+\alpha^{\left\lceil\frac{k-K}{\ell}\right\rceil}(k-K+j) \Delta_{\alpha(\ell)} u(K+j) \text { for all } k \in[K, \infty),
$$

where $j=\frac{k-K}{\ell}-\left[\frac{k-K}{\ell}\right] \ell$. So,

Proof. Since $\Delta_{\alpha(\ell)}^{2} u(k) \geq 0$ for all $k \in[K, \infty), \Delta_{\alpha(\ell)} u(k)$ is nondecreasing.

$$
u(k)-\alpha^{\left\lceil\frac{k-K}{\ell}\right\rceil} u(K+j)=\sum_{r=0}^{\frac{k-\ell-K-j}{\ell}} \frac{\Delta_{\alpha(\ell)} u(K+j+r \ell)}{\alpha^{\left\lceil\frac{K-k+j+\ell+r \ell}{\ell}\right\rceil}}
$$




$$
\geq(k-K-j) \Delta_{\alpha(\ell)} u(K+j),
$$

where $j=\frac{k-K}{\ell}-\left[\frac{k-K}{\ell}\right] \ell$.

Lemma 3.2. If $u(k)$ is a nontrivial real solution of (1) and if

$$
\begin{array}{cl}
\text { (a) } u(k) \geq 0, & \text { (b) } \Delta_{\alpha(\ell)} u(k) \geq 0, \\
\text { (c) } \Delta_{\alpha(\ell)}^{2} u(k-\ell) \geq 0, \quad & \text { (d) } \Delta_{\alpha(\ell)}^{3} u(k-2 \ell) \geq 0
\end{array}
$$

for some $k=a+j, a \in[2 \ell, \infty)$, for all $j=[0, \ell)$ then (12) holds for all $k \in[a+\ell, \infty)$, with the strict inequality in (12a) for all $k \in[a+3 \ell, \infty)$, strict inequality in $(12 b)$ for all $k \in[a+2 \ell, \infty)$ and strict inequality in (12c) and (12d) for all $k \in[a+4 \ell, \infty)$. Furthermore

$$
\Delta_{\alpha(\ell)}^{4} u(k-2 \ell) \geq 0, \text { for all } k \in[a, \infty),
$$

with the strict inequality for all $k \in[a+2 \ell, \infty)$ and $u(k), \Delta_{\alpha(\ell)} u(k)$ and $\Delta_{\alpha(\ell)}^{2} u(k)$ all tend to $+\infty$ as $k \rightarrow \infty$.

Proof. Since $u(a+j) \geq 0, \Delta_{\alpha(\ell)} u(a+j) \geq 0, \Delta_{\alpha(\ell)}^{2} u(a+j-\ell) \geq 0$ and $\Delta_{\alpha(\ell)}^{3} u(a+j-2 \ell) \geq 0$, we immediately have $u(a+j+\ell)-\alpha u(k+j)=$ $\Delta_{\alpha(\ell)} u(a+j) \geq 0$, hence

$$
u(a+j+\ell) \geq \alpha u(a+j) \geq 0 .
$$

The fourth order generalized difference equation (6) can be written in the form

$$
\begin{array}{r}
\Delta_{\alpha(\ell)}^{4} u(a+j-2 \ell)=u(a+j+2 \ell)-\alpha u(a+j+\ell)-\alpha \Delta_{\alpha(\ell)} u(a+j) \\
-\alpha \Delta_{\alpha(\ell)}^{2} u(a+j-\ell)-\alpha \Delta_{\alpha(\ell)}^{3} u(a+j-2 \ell)
\end{array}
$$

and by (1) we obtain

$$
\begin{gathered}
u(a+j+2 \ell)=\alpha \Delta_{\alpha(\ell)}^{3} u(a+j-2 \ell)+\alpha \Delta_{\alpha(\ell)}^{2} u(a+j-\ell) \\
+\alpha \Delta_{\alpha(\ell)} u(a+j)+\alpha u(a+j+\ell)+\alpha^{2} p(a+j) u(a+j) . \\
\Delta_{\alpha(\ell)} u(a+j+\ell)=u(a+j+2 \ell)-\alpha u(a+j+\ell)=\alpha \Delta_{\alpha(\ell)}^{3} u(a+j-2 \ell) \\
+\alpha \Delta_{\alpha(\ell)}^{2} u(a+j-\ell)+\alpha \Delta_{\alpha(\ell)} u(a+j)+\alpha^{2} p(a+j) u(a+j) .
\end{gathered}
$$

Then (15) yields

$$
\begin{aligned}
& \Delta_{\alpha(\ell)}^{2} u(a+j)=\Delta_{\alpha(\ell)} u(a+j+\ell)-\alpha \Delta_{\alpha(\ell)} u(a+j) \\
& \quad=\alpha \Delta_{\alpha(\ell)}^{3} u(a+j-2 \ell)+\alpha \Delta_{\alpha(\ell)}^{2} u(a+j-\ell)+\alpha^{2} p(a+j) u(a+j),
\end{aligned}
$$


and this in turn leads to

$$
\begin{aligned}
\Delta_{\alpha(\ell)}^{3} u(a+j-\ell) & =\Delta_{\alpha(\ell)}^{2} u(a+j)-\alpha \Delta_{\alpha(\ell)}^{2} u(a+j-\ell) \\
& =\alpha \Delta_{\alpha(\ell)}^{3} u(a+j-2 \ell)+\alpha^{2} p(a+j) u(a+j) .
\end{aligned}
$$

All the terms on the right-hand sides of (15), (16) and (17) are nonnegative, so $\Delta_{\alpha(\ell)} u(a+j+\ell), \Delta_{\alpha(\ell)}^{2} u(a+j)$ and $\Delta_{\alpha(\ell)}^{3} u(a+j-\ell)$ are nonnegative. Thus, assuming that conditions (12a-d) hold for $a+j$ implies that they also hold for $a+j+\ell$. Proceeding in this way, we see by mathematical induction that conditions (12a-d) hold for all $k \in[a, \infty)$. Condition (13) follows immediately from $(1)$, since $p(k+j)>0$ and $u(k+j) \geq 0$ for all $k \geq[a+\ell, \infty)$.

Since $p(a+j)>0$, at least one term on the right in (15) must be positive, since otherwise $u(a+j+\ell)=u(a+j)=u(a+j-\ell)=u(a+j-2 \ell)=0$ by (6), hence $u(a+j)$ would be the trivial solution of (1) by Lemma 2.6. Thus $\Delta_{\alpha(\ell)} u(a+j+\ell)>0$ and it follows that the strict inequality holds in (12b) for all $k \in[a+\ell, \infty)$. This implies that $u(k)$ is strictly increasing for $k \in[a+\ell, \infty)$ and since $u(a+j+\ell) \geq 0$, we then have $u(k)>0$ for all $k \in[a+2 \ell, \infty)$, as claimed. This in turn implies, by use of (1), that strict inequality holds for (13) for $k \in[a+2 \ell, \infty)$. Also, using (16) and (17), we may conclude that $\Delta_{\alpha(\ell)}^{2} u(k)>0$ and $\Delta_{\alpha(\ell)}^{3} u(k-\ell)>0$ for all $k \in[a+2 \ell, \infty)$. Therefore, the strict inequality holds in (12c) and (12d) for all $k \in[a+3 \ell, \infty)$.

To prove the last statement in the conclusion, we observe that the functions $u(k), v(k)=\Delta_{\alpha(\ell)} u(k)$ and $w(k)=\Delta_{\alpha(\ell)}^{2} u(k)$ all satisfy the hypotheses of Lemma 3.1 for any $K \in[a, \infty)$. Application of Lemma 3.1 to these three sequences yields, respectively,

$$
\begin{gathered}
u(k) \geq \alpha^{\left\lceil\frac{k-K}{\ell}\right\rceil} u(K+j)+\Delta_{\alpha(\ell)} u(K+j)(k-K-j), \\
\Delta_{\alpha(\ell)} u(k) \geq \Delta_{\alpha(\ell)} u(K+j) \alpha^{\left\lceil\frac{k-K}{\ell}\right\rceil}+\Delta_{\alpha(\ell)}^{2} u(K+j)(k-K-j), \\
\Delta_{\alpha(\ell)}^{2} u(k) \geq \Delta_{\alpha(\ell)}^{2} u(K+j) \alpha^{\left\lceil\frac{k-K}{\ell}\right\rceil}+\Delta_{\alpha(\ell)}^{3} u(K+j)(k-K-j),
\end{gathered}
$$

where $j=\frac{k-K}{\ell}-\left[\frac{k-K}{\ell}\right] \ell$ for all $k \geq K$. In particular, these inequalities are true for $K=a+2 \ell$ and since $\Delta_{\alpha(\ell)} u(K+j), \Delta_{\alpha(\ell)}^{2} u(K+j)$ and $\Delta_{\alpha(\ell)}^{3} u(K+j)$ are all positive for $K=a+2 \ell$, it follows that $u(k), \Delta_{\alpha(\ell)} u(k)$ and $\Delta_{\alpha(\ell)}^{2} u(k)$ tend to $+\infty$ as $k \rightarrow \infty$, which completes the proof.

Remark 3.3. The above Lemma 3.2 fails if the hypothesis $u(a) \geq 0$ is replaced by $u(a+\ell) \geq 0$. This is shown by the example $u(k)=(-\alpha)^{\left[\frac{k+\ell}{\ell}\right]}$, which is a solution of $\Delta_{\alpha(\ell)}^{4} u(k-2 \ell)=16 \alpha^{2} u(k)$. Let $\ell=0.5$ and $k=1.7$. Then the 
function satisfies $u(1.7)=\alpha^{4}>0, \Delta_{\alpha(\ell)} u(1.2)=2 \alpha^{4}>0, \Delta_{\alpha(\ell)}^{2} u(0.7)=4 \alpha^{4}>$ $0, \Delta_{\alpha(\ell)}^{3} u(0.2)=8 \alpha^{4}>0$, but fails to have any of the monotonicity properties of the conclusion of Lemma 3.2. It is interesting to note also that the initial conditions given in the hypotheses of Lemma 3.2 are not necessarily of "convex" type, i.e., the graph of a solution satisfying these conditions is not necessarily convex upward in the interval from $[a-2 \ell, a+\ell]$ for $a \in[2 \ell, \infty)$. For example, a function $u(k)$ satisfying $u(0)=0, u(\ell)=\ell, u(2 \ell)=0, u(3 \ell)=\ell$, satisfies the hypotheses of Lemma 3.2, since $u(2 \ell)=0, \Delta_{\alpha(\ell)} u(2 \ell)=\ell>0, \Delta_{\alpha(\ell)}^{2} u(\ell)=2 \ell>$ 0 and $\Delta_{\alpha(\ell)}^{3} u(0)=4 \ell>0$. However, the "convex" conditions $u(0)=2 \ell, u(\ell)=$ $0, u(2 \ell)=0, u(3 \ell)=\ell$, do not, since in this case $u(2 \ell)=0, \Delta_{\alpha(\ell)} u(2 \ell)=$ $\ell, \Delta_{\alpha(\ell)}^{2} u(\ell)=\ell$, but $\Delta_{\alpha(\ell)}^{3} u(0)=-\ell$.

Lemma 3.4. If $u(k)$ is a nontrivial solution of (1) and if

$$
\begin{array}{cc}
\text { (a) } u(k) \geq 0, & \text { (b) } \Delta_{\alpha(\ell)} u(k) \geq 0, \\
\text { (c) } \Delta_{\alpha(\ell)}^{2} u(k) \geq 0, & \text { (d) } \Delta_{\alpha(\ell)}^{3} u(k) \geq 0
\end{array}
$$

for some interval $k \in[a, a+\ell)$ for some $a \in[0, \infty)$, then (18) holds for all $k \in[a, \infty)$, with the strict inequality in $(18 \mathrm{a}, b, d)$ for all $k \in[a+3 \ell, \infty)$ and in (18c) for all $k \in[a+4 \ell, \infty)$. Furthermore,

$$
\Delta_{\alpha(\ell)}^{4} u(k) \geq 0 \text { for all } k \in[a, \infty)
$$

with the strict inequality for all $k \in[a+2 \ell, \infty)$ and $u(k), \Delta_{\alpha(\ell)} u(k)$ and $\Delta_{\alpha(\ell)}^{2} u(k)$ all tend to $\infty$ as $k \rightarrow \infty$.

Proof. Given a nontrivial solution $u(k)$ of (1) satisfying (19) for some $k \in$ $[a, a+\ell), a \in[0, \infty)$, let $k_{1}=a+2 \ell$. Then $\Delta_{\alpha(\ell)}^{3} u\left(k_{1}+j-2 \ell\right)=\Delta_{\alpha(\ell)}^{2} u\left(k_{1}+\right.$ $j-\ell)-\alpha \Delta_{\alpha(\ell)}^{2} u\left(k_{1}+j-2 \ell\right) \geq 0$, so

$$
\Delta_{\alpha(\ell)}^{2} u\left(k_{1}+j-\ell\right) \geq \Delta_{\alpha(\ell)}^{2} u\left(k_{1}+j-2 \ell\right)=\Delta_{\alpha(\ell)}^{2} u(a+j) \geq 0 .
$$

Similarly, $\Delta_{\alpha(\ell)}^{2} u\left(k_{1}-2 \ell\right) \geq 0$ implies $\Delta_{\alpha(\ell)} u\left(k_{1}-\ell\right) \geq \Delta_{\alpha(\ell)} u\left(k_{1}-2 \ell\right) \geq 0$ and $\Delta_{\alpha(\ell)}^{2} u\left(k_{1}+j-\ell\right) \geq 0$ implies $\Delta_{\alpha(\ell)} u\left(k_{1}+j\right) \geq \Delta_{\alpha(\ell)} u\left(k_{1}+j-\ell\right)$, hence

$$
\Delta_{\alpha(\ell)} u\left(k_{1}+j\right) \geq \Delta_{\alpha(\ell)} u\left(k_{1}+j-\ell\right) \geq \Delta_{\alpha(\ell)} u\left(k_{1}+j-2 \ell\right) \geq 0 .
$$

This in turn implies

$$
u\left(k_{1}+j+\ell\right) \geq u\left(k_{1}+j\right) \geq u\left(k_{1}+j-\ell\right) \geq u\left(k_{1}+j-2 \ell\right) \geq 0 .
$$


Thus $\Delta_{\alpha(\ell)}^{3} u\left(k_{1}+j-2 \ell\right), \Delta_{\alpha(\ell)}^{2} u\left(k_{1}+j-\ell\right), \Delta_{\alpha(\ell)} u\left(k_{1}+j\right)$ and $u\left(k_{1}+j\right)$ are all nonnegative and it follows from Lemma 3.2 that

$$
\begin{array}{rlrl}
(a) u(k) \geq 0, & (b) \Delta_{\alpha(\ell)} u(k) \geq 0, & (c) \Delta_{\alpha(\ell)}^{2} u(k-\ell) & \geq 0, \\
(d) \Delta_{\alpha(\ell)}^{3} u(k-2 \ell) \geq 0, & (e) \Delta_{\alpha(\ell)}^{4} u(k-2 \ell) \geq 0,
\end{array}
$$

for all $k \in\left[k_{1}, \infty\right)$, with the strict inequality in (22a, e) for $k \in\left[k_{1}+2 \ell, \infty\right)$, in (22b) for $k \in\left[k_{1}+\ell, \infty\right)$ and in (22c, d) for $k \in\left[k_{1}+3 \ell, \infty\right)$. Also, $u(k), \Delta_{\alpha(\ell)} u(k)$ and $\Delta_{\alpha(\ell)}^{2} u(k)$ tend to $\infty$ as $k \rightarrow \infty$. By shifting subscripts and using $k_{1}=$ $a+2 \ell$, we may rewrite (22) and the related statements about the strict inequality as follows:

$$
\begin{array}{ll}
(a) u(k) \geq 0, k \in[a+2 \ell, \infty) & (>0 \text { for } k \in[a+4 \ell, \infty)), \\
(b) \Delta_{\alpha(\ell)} u(k) \geq 0, k \in[a+2 \ell, \infty) & (>0 \text { for } k \in[a+3 \ell, \infty)), \\
(c) \Delta_{\alpha(\ell)}^{2} u(k) \geq 0, k \in[a+\ell, \infty) & (>0 \text { for } k \in[a+4 \ell, \infty)), \\
(d) \Delta_{\alpha(\ell)}^{3} u(k) \geq 0, k \in[a, \infty) & (>0 \text { for } k \in[a+3 \ell, \infty)), \\
(e) \Delta_{\alpha(\ell)}^{4} u(k) \geq 0, k \in[a, \infty) & (>0 \text { for } k \in[a+2 \ell, \infty)) .
\end{array}
$$

Since $k_{1}=a+2 \ell,(20)$ and (21) immediately imply that $u(k) \geq 0$ and $\Delta_{\alpha(\ell)} u(k) \geq 0$ hold also for $k=a+\ell$ and $k=a$. Furthermore, (21) implies that if $u(a+j+3 \ell)=0$, then $u(a+j+3 \ell)=u(a+j+2 \ell)=u(a+j+\ell)=u(a+j)=0$, so $u(k)$ would be the trivial solution of (1). Therefore, $u(a+j+3 \ell)>0$. Finally, $\Delta_{\alpha(\ell)}^{2} u(a+j) \geq 0$ by hypothesis and we have shown that all conditions of (18) hold for $k \geq a$, with the strict inequality as stated in the conclusion.

Lemma 3.5. If $u(k)$ is a nontrivial solution of (1) with

$$
\begin{array}{ll}
\text { (a) } u(k) \geq 0, & \text { (b) } \Delta_{\alpha(\ell)} u(k-\ell) \leq 0, \\
\text { (c) } \Delta_{\alpha(\ell)}^{2} u(k-\ell) \geq 0, & \text { (d) } \Delta_{\alpha(\ell)}^{3} u(k-\ell) \leq 0
\end{array}
$$

for some interval $k \in[a, a+\ell), a \in[3 \ell, \infty)$, then (24) holds for all $k \in[2 \ell, a]$ and

$$
\Delta_{\alpha(\ell)}^{4} u(k-2 \ell) \geq 0 \text { for all } k \in[2 \ell, a] .
$$

Furthermore, $u(0)>u(1)>0$ and $\Delta_{\alpha(\ell)} u(0)<0$. Strict inequality holds in (24a) and (25) for all $k \in[2 \ell, a-2 \ell]$ if $a \in[4 \ell, \infty)$, in (24b) for all $k \in[2 \ell, a-\ell]$ and in $(24 c, d)$ for all $k \in[2 \ell, a-3 \ell]$ if $a \in[5 \ell, \infty)$. 
Proof. Let $u(k)$ be a nontrivial solution of (1) satisfying (24) for some interval $k \in[a, a+\ell), a \in[3 \ell, \infty)$. Let

$$
v(i)=u(2 a+j-i), i \in[0,2 a] .
$$

Then it is readily verified that

$$
\begin{aligned}
\Delta_{\alpha(\ell)} v(i) & =-\Delta_{\alpha(\ell)} u(2 a-i-\ell), & & i \in[0,2 a-\ell], \\
\Delta_{\alpha(\ell)}^{2} v(i) & =\Delta_{\alpha(\ell)}^{2} u(2 a-i-2 \ell), & & i \in[0,2 a-2 \ell], \\
\Delta_{\alpha(\ell)}^{3} v(i) & =-\Delta_{\alpha(\ell)}^{3} u(2 a-i-3 \ell), & & i \in[0,2 a-3 \ell], \\
\Delta_{\alpha(\ell)}^{4} v(i) & =\Delta_{\alpha(\ell)}^{4} u(2 a-i-4 \ell), & & i \in[0,2 a-4 \ell] .
\end{aligned}
$$

In particular, since $u(k)$ is a solution of $(1)$,

$$
\Delta_{\alpha(\ell)}^{4} v(i-2 \ell)=\Delta_{\alpha(\ell)}^{4} u(2 a+j-i-2 \ell)=\alpha^{2} p(2 a+j-i) u(2 a+j-i),
$$

$i \in[2 \ell, 2 a-2 \ell]$. So $v(k)$ is a solution of

$$
\Delta_{\alpha(\ell)}^{4} v(i-2 \ell)=P(i) v(i), \quad i \in[2 \ell, 2 a-2 \ell],
$$

where $P(i)=p(2 a-i)>0, i \in[2 \ell, 2 a-2 \ell]$. Using (26) and the definition of $v(i)$, we find that $v(a+j)=u(a+j), \Delta_{\alpha(\ell)} v(a+j)=-\Delta_{\alpha(\ell)} u(a+j-$ $\ell), \Delta_{\alpha(\ell)}^{2} u(a+j-\ell)$ and $\Delta_{\alpha(\ell)}^{3} v(a+j-2 \ell)=-\Delta_{\alpha(\ell)}^{3} u(a+j-\ell)$. It then follows from our hypotheses that $v$ satisfies the hypotheses of Lemma 3.2, which implies that
(a) $v(i) \geq 0$
(b) $\Delta_{\alpha(\ell)} v(i) \geq 0$,
(c) $\Delta_{\alpha(\ell)}^{2} v(i-\ell) \geq 0$
(d) $\Delta_{\alpha(\ell)}^{3} v(i-2 \ell) \geq 0$,
(e) $\Delta_{\alpha(\ell)}^{4} v(i-2 \ell) \geq 0$,

for $a \leq i \leq 2 a-2 \ell$, with the strict inequality holding as follows:
(a) $v(i)>0$,
$i \in[a+2 \ell, 2 a-2 \ell](a \in[4 \ell, \infty))$,
(b) $\Delta_{\alpha(\ell)} v(i)>0$,
$i \in[a+\ell, 2 a-2 \ell](a \in[3 \ell, \infty))$,
(c) $\Delta_{\alpha(\ell)}^{2} v(i-\ell)>0$,
$i \in[a+3 \ell, 2 a-2 \ell](a \in[5 \ell, \infty))$,
(d) $\Delta_{\alpha(\ell)}^{3} v(i-2 \ell)>0$,
$i \in[a+3 \ell, 2 a-2 \ell](a \in[5 \ell, \infty))$,
(e) $\Delta_{\alpha(\ell)}^{4} v(i-2 \ell)>0$,
$i \in[a+2 \ell, 2 a-2 \ell](a \in[4 \ell, \infty))$.

It follows from (26) and (28) that $(a) u(2 a+j-i) \geq 0$,

(b) $\Delta_{\alpha(\ell)} u(2 a+j-i-\ell) \leq 0,(c) \Delta_{\alpha(\ell)}^{2} u(2 a+j-i-\ell) \geq 0$, 


$$
\text { (d) } \Delta_{\alpha(\ell)}^{3} u(2 a+j-i-\ell) \leq 0, \quad \text { (e) } \Delta_{\alpha(\ell)}^{4} u(2 a+j-i-2 \ell) \geq 0
$$

for $i \in[a, 2 a-2 \ell]$, with the strict inequality for the same values of $i$ as given in (29). If we replace $k=2 a+j-i$, we may rewrite (30) as

$$
\begin{aligned}
(a) u(k) \geq 0, \quad(b) \Delta_{\alpha(\ell)} u(k-\ell) \leq 0, & (c) \Delta_{\alpha(\ell)}^{2} u(k-\ell) \geq 0, \\
(d) \Delta_{\alpha(\ell)}^{3} u(k-\ell) \leq 0, & (e) \Delta_{\alpha(\ell)}^{4} u(k-2 \ell) \geq 0,
\end{aligned}
$$

for $k \in[2 \ell, a]$, which proves that (24) holds as claimed. The values of $k$ which give the strict inequality in (31), as claimed the conclusions of the lemma, follow immediately by letting, $i=2 a+j-k$ in the $i$-intervals stated in (29). To complete the proof we need to extend the domain of (31a, b) by showing $u(0)>u(1)>0$ and $\Delta_{\alpha(\ell)} u(0)<0$. For this, we first observe that $(29 \mathrm{~b})$ implies $v(2 a+j-\ell)-\alpha v(2 a+j-2 \ell)=\Delta_{\alpha(\ell)} v(2 a+j-2 \ell)>0$ so

$$
u(\ell)=v(2 a+j-\ell)>v(2 a+j-2 \ell) \geq 0 .
$$

Also, $v$ is a solution of (27) and we may rewrite (27), as in (14), as

$$
\begin{aligned}
v(i+2 \ell)=\alpha \Delta_{\alpha(\ell)}^{3} v & (i-2 \ell)+\alpha \Delta_{\alpha(\ell)}^{2} v(i-\ell) \\
& +\alpha \Delta_{\alpha(\ell)} v(i)+v(i+\ell)+\alpha^{2} P(i) v(i) .
\end{aligned}
$$

In particular,

$$
\begin{aligned}
& v(2 a+j)=\alpha \Delta_{\alpha(\ell)}^{3} v(2 a+j-4 \ell)+\alpha \Delta_{\alpha(\ell)}^{2} v(2 a+j-3 \ell)+ \\
& \alpha \Delta_{\alpha(\ell)} v(2 a+j-2 \ell)+v(2 a+j-\ell)+\alpha^{2} P(2 a+j-2 \ell) v(2 a+j-2 \ell)
\end{aligned}
$$

from which it follows by $(28 \mathrm{~b}, \mathrm{c}, \mathrm{d})$ with $i=2 a+j-2 \ell$ and by $(32)$, that

$$
u(0)=v(2 a)>0 .
$$

Similarly (28) and (34) imply

$$
\begin{aligned}
& \Delta_{\alpha(\ell)} v(2 a+j-\ell)=v(2 a+j)-\alpha v(2 a+j-\ell)=\alpha \Delta_{\alpha(\ell)}^{3} v(2 a+j-4 \ell) \\
& +\alpha \Delta_{\alpha(\ell)}^{2} v(2 a+j-3 \ell)+\alpha \Delta_{\alpha(\ell)} v(2 a+j-2) \\
& +\alpha^{2} P(2 a+j-2 \ell) v(2 a+j-2)>0
\end{aligned}
$$

hence $\Delta_{\alpha(\ell)} u(0)=-\Delta_{\alpha(\ell)} v(2 a+j-\ell)<0$, which yields $u(\ell)<u(0)$, which completes the proof. 
Lemma 3.6. Let $a \in[2 \ell, \infty)$. If $u(k)$ is a solution of (1) with $u(a+j)=$ $0, u(a+j-\ell) \geq 0, u(a+j+\ell) \geq 0, u(a+j-\ell)$ and $u(a+j+\ell)$ for all $j \in[0, \ell)$ not both 0 , then at least one of the following conditions must be true.

(a) Either $u(k)>0$ for all $k \in[a+\ell, \infty)$,or

(b) $u(k)>0$ for all $k \in(-\infty, a-\ell], a \in[0, \infty)$.

In particular, $u(k)$ cannot have $\ell$-generalized zeros of any order at both $R$ and $S$, where $R<a+j-\ell<a+j+\ell<S$. An analogous statement holds for the hypotheses $u(a+j-\ell) \leq 0$ and $u(a+j+\ell) \leq 0$.

Proof. From the hypotheses, we have

$$
\begin{gathered}
u(a+j)=0, \\
\Delta_{\alpha(\ell)} u(a+j)=u(a+j+\ell)-\alpha u(a+j)=u(a+j+\ell) \geq 0, \\
\Delta_{\alpha(\ell)} u(a+j-\ell)=u(a+j)-\alpha u(a+j-\ell)=-\alpha u(a+j-\ell) \leq 0, \\
\Delta_{\alpha(\ell)}^{2} u(a+j-\ell)=u(a+j+\ell)-2 \alpha u(a+j)+\alpha^{2} u(a+j-\ell)>0 .
\end{gathered}
$$

If $\Delta_{\alpha(\ell)}^{3} u(a+j-2 \ell) \geq 0$, then Lemma 3.2 holds and we can conclude $u(k)>0$ for all $k \in[a+2 \ell, \infty)$. On the other hand, if $\Delta_{\alpha(\ell)}^{3} u(a+j-2 \ell)<0$, then (1) implies $\Delta_{\alpha(\ell)}^{3} u(a+j-\ell)-\Delta_{\alpha(\ell)}^{3} u(a+j-2 \ell)=\Delta_{\alpha(\ell)}^{4} u(a+j-2 \ell)=p(a+j) u(a+j)=0$, hence $\Delta_{\alpha(\ell)}^{3} u(a+j-\ell)=\Delta_{\alpha(\ell)}^{3} u(a+j-2 \ell)>0$. If $a \in[3 \ell, \infty)$, then the hypotheses of Lemma 3.5 are satisfied, hence $u(k)>0$ for all $k \in[a-2 \ell, \infty)$. If $a=2 \ell$, then $\Delta_{\alpha(\ell)}^{3} u(a+j-2 \ell)=u(3 \ell)-3 \alpha u(2 \ell)+3 \alpha^{2} u(\ell)-\alpha^{3} u(0)$. Since $u(a+j)=u(2 \ell)=0$ and $\Delta_{\alpha(\ell)}^{3} u(a+j-2 \ell)=\Delta_{\alpha(\ell)}^{3} u(a+j-\ell)<0$, we have that $u(3 \ell)+3 \alpha^{2} u(\ell)<\alpha^{3} u(0)$. Since $u(3 \ell)$ and $u(\ell)$ are nonnegative and are not both equal to zero, it must be true that $u(0)>0$, which is part (b) of our conclusion for $a=2 \ell$.

\section{Separation Theorems}

Theorem 4.1. If $u(k)$ is a nontrivial solution of (1) with zeros at three consecutive values of $k$, say $a+j, a+j+\ell$ and $a+j+2 \ell$ for all $j=[0, \ell)$, then $u(k)$ has no other $\ell$-generalized zeros. If $u(a+j+3 \ell)>0(<0)$, then $\Delta_{\alpha(\ell)} u(k) \geq 0(\leq 0)$ for all $k$ and the inequality is strict if $k \in[a+2 \ell, \infty)$ or $k \in[0, a-\ell]$. In particular, if $\gamma \in[0, a-\ell]$ and $\beta \in[0, a+3 \ell]$, then $u(\gamma) u(\beta)<0$. 
Proof. Clearly $\Delta_{\alpha(\ell)} u(a+j)=\Delta_{\alpha(\ell)}^{2} u(a+j)=0$. Since the solution $u(k)$ is nontrival, we may assume that $u(a+j+3 \ell)>0$. Thus, $\Delta_{\alpha(\ell)}^{3} u(a+j)=$ $u(a+j+3 \ell)>0$ and by Lemma 3.4, $u(k)$ is positive and strictly increasing for $k \in[a+3 \ell, \infty)$. Let $v(k)=-u(k)$. Then, $v(a+j+\ell)=0, \Delta_{\alpha(\ell)} v(a+j)=$ $0, \Delta_{\alpha(\ell)}^{2} v(a+j)=0$ and $\Delta_{\alpha(\ell)}^{3} v(a+j)<0$. If $a \in[2 \ell, \infty)$, then Lemma 3.5 implies that $v(k)$ is positive and strictly decreasing on $[0, a]$. Thus, $u(k)$ is negative and strictly increasing on $[0, a]$. If $a=\ell$, then we again assume that $u(a+j+3 \ell)=u(4 \ell)>0$. Then, by $(1), \Delta_{\alpha(\ell)}^{4} u(0)=\alpha^{2} p(2 \ell) u(2 \ell)=0$. But, $\Delta_{\alpha(\ell)}^{4} u(0)=u(4 \ell)+\alpha^{4} u(0)$ by $(6)$, so $u(0)=-u(4 \ell)<0$ and $\Delta_{\alpha(\ell)} u(0)=$ $\alpha^{3} u(\ell)-\alpha^{4} u(0)>0$, as claimed. If $a=0$, then the part of the conclusion concerning $k \leq a-\ell$ is empty. This completes the proof.

Theorem 4.2. Let $a \in[\ell, \infty)$ and suppose that $u(k)$ is a solution of (1) with $u(a+j)=0, u(a+j+\ell)=0, u(a+j+2 \ell) \neq 0$ for all $j=[0, \ell)$, but $a+2 \ell$ is a $\ell$-generalized zero for $u(k)$. Then, $u(k)$ has no other $\ell$-generalized zeros. If $u(a+j+2 \ell)>0(<0)$, then $\Delta_{\alpha(\ell)} u(k) \geq(\leq 0)$ for all $k \in[0, \infty)$, with the strict inequality for all $k \in[a+2 \ell, \infty)$ or $k \in[0, a-\ell]$. In particular, if $\gamma \in[0, a-\ell]$ and $\beta \in[a+2 \ell, \infty)$, then $u(\gamma) u(\beta)<0$.

Proof. Since $u(a+j+2 \ell) \neq 0$, we can assume that $u(a+j+2 \ell)>0$. Since $u(a+j)=u(a+j+\ell)=0, a+j+2 \ell$ cannot be a $\ell$-generalized zero of order 1 or 2 and Theorem 4.1 implies that the order cannot be greater than 3 . Thus, $a+j+2 \ell$ is a $\ell$-generalized zero of order 3 , which implies that $u(a+j-\ell)<0$. Now, since from (1), we have

$$
\begin{aligned}
& u(a+j+3 \ell)-4 \alpha u(a+j+2 \ell)+6 \alpha^{2} u(a+j+\ell) \\
& -4 \alpha^{3} u(a+j)+\alpha^{4} u(a+j-\ell)=\alpha^{2} p(a+j+\ell) u(a+j+\ell), \\
& \text { or } u(a+j+3 \ell)=4 \alpha u(a+j+2 \ell)-\alpha^{2} u(a+j-\ell),
\end{aligned}
$$

it follows that

$$
\begin{array}{r}
\Delta_{\alpha(\ell)}^{3} u(a+j)=u(a+j+3 \ell)-3 \alpha u(a+j+2 \ell)+3 \alpha^{2} u(a+j+\ell)-\alpha^{3} u(a+j) \\
\quad=4 \alpha u(a+j+2 \ell)-u(a+j-\ell)-3 \alpha u(a+j+2 \ell)+3 \alpha^{2} u(a+j+\ell)-\alpha^{3} u(a+ \\
j)=\alpha u(a+j+2 \ell)-u(a+j-\ell)>0 . \text { Clearly, } \Delta_{\alpha(\ell)}^{2} u(a+j)>0, \Delta_{\alpha(\ell)} u(a+j)=0
\end{array}
$$
and $u(a+j)=0$, thus by Lemma 3.4,u(k) is positive and strictly increasing on $[a+3 \ell, \infty)$. For $k \in[0, a]$, let $v(k)=-u(k)$. Then, $v(a+j)=0, \Delta_{\alpha}(\ell) v(a+$ $j-\ell)<0, \Delta_{\alpha(\ell)}^{2} v(a+j-\ell)>0$ and $\Delta_{\alpha(\ell)}^{3} v(a+j-\ell)<0$. If $a \in[3 \ell, \infty)$, then the result follows as in Theorem 4.1 and Lemma 3.5. If $a=2 \ell$, then $u(2 \ell)=u(3 \ell)=0, u(\ell)<0, u(4 \ell)>0$ and $\Delta_{\alpha(\ell)} u(\ell)>0$. By $(1)$, we have $\Delta_{\alpha(\ell)}^{4} u(0)=\alpha^{2} p(2 \ell) u(2 \ell)=0$. But, $\Delta_{\alpha(\ell)}^{4} u(0)=u(4 \ell)-4 \alpha u(3 \ell)+6 \alpha^{2} u(2 \ell)-$ 
$4 \alpha^{3} u(\ell)+\alpha^{4} u(0)=u(4 \ell)-4 \alpha^{3} u(\ell)+\alpha^{4} u(0)$ and so $4 u(\ell)-\alpha^{4} u(0)=u(4 \ell)>0$. Hence, $u(0)<4 u(\ell)<0$ and $u(0)-u(\ell)<3 u(\ell)<0$. Therefore, $u(0)<0$ and $\Delta_{\alpha(\ell)} u(0)>0$, as claimed. If $a=\ell$, then $u(\ell)=u(2 \ell)=0, u(3 \ell) \neq 0$ and $a+2 \ell=3 \ell$ is a $\ell$-generalized zero. It follows from the definition of a $\ell$-generalized zero that this must be a $\ell$-generalized zero of order 3 . So, if $u(3 \ell)>0$ then $u(0)<0$. Hence, $\Delta_{\alpha(\ell)} u(0)>0$, which completes the proof.

Corollary 4.3. If $u(k)$ is a nontrivial solution of (1) with $\ell$-generalized zeros at $\gamma$ and $\beta$ and a zero at $a+j$, where $\gamma+\ell<a+j<\beta-\ell$, then $u(a+j-\ell) u(a+j+\ell)<0$. In particular, $u(k)$ does not have a $\ell$-generalized zero at $a+j+\ell$.

Proof. Since $\gamma+\ell<a+j<\beta-\ell$, from Theorem 4.1 it follows that $u(a+j+\ell)$ and $u(a+j-\ell)$ both cannot be zero. If $u(a+j+\ell) u(a+j-\ell) \geq 0$, then Lemma 3.6 implies that $u(k)$ cannot have $\ell$-generalized zeros at both $\gamma$ and $\beta$, which is a contradiction. Thus, $u(a+j-\ell) u(a+j+\ell)<0$.

Corollary 4.4. If $u(k)$ is a nontrivial solution of (1) with $u(\gamma)=u(a+j)=$ $u(\beta)=0$, where $\gamma<a+j<\beta-\ell$, then $u(a+j+\ell) \neq 0$.

Proof. If $a+j=\gamma+\ell$, the corollary follows immediately from Theorem 4.1. If $a+j>\gamma+\ell$, it follows from Corollary 4.3.

Corollary 4.5. If a nontrivial solution $u(k)$ of (1) has a zero at $\gamma$ and a $\ell$-generalized zero at $\beta$, where $\gamma<\beta$, then $u(k)$ cannot have consecutive zeros at $a+j, a+j+\ell$ where $\gamma<a+j<\beta-\ell$.

Proof. Let $u(k)$ be a nontrivial solution of (1) with zeros at $\gamma, a+j, a+j+\ell$ for all $j=[0, \ell)$ and a $\ell$-generalized zero at $\beta$, where $\gamma<a+j<\beta \ell$. Theorems 4.1 and 4.2 imply that we must have $\gamma<a+j-\ell$ and $\beta>a+j+2 \ell$, i.e., $\gamma+\ell<a+j<\beta-\ell$. Corollary 4.3 implies $u(a+j-\ell) u(a+j+\ell)<0$, contradicting $u(a+j+\ell)=0$.

Remark 4.6. Corollary 4.5 says that, if a solution $u(k)$ of $(1)$ has four or more zeros, then no two zeros can occur at consecutive values of $k$, unless they are the first two zeros or the last two zeros. For example, consider the function $u(k)=\{-4 \ell, 0,0,-\ell, 0, \ell, 0,0,4 \ell, 15 \ell, \ldots\}$ which is a solution of $\Delta_{\alpha(\ell)}^{4} u(k-$ $2 \ell)=5 u(k)$ with $u(\ell)=-4 \ell$. This solution is positive and increasing for all $k \in[9 \ell, \infty)$ follows from Lemma 3.2 with $a=7 \ell$. Also, the terms $u(3 \ell)$ through $u(7 \ell)$ illustrate Corollary 4.3. 
Theorem 4.7. If two nontrivial solutions $u(k)$ and $v(k)$ of (1) have three zeros in common, then $u(k)$ and $v(k)$ are linearly dependent, i.e. specifying any three zeros uniquely determines a nontrivial solution up to a multiplicative constant.

Proof. If $u(\gamma)=u(a+j)=u(a+j+\ell)=v(\gamma)=v(a+j)=v(a+j+\ell)=0$ for all $j \in[0, \ell)$, for some $\gamma$ and $a$, where $0 \leq \gamma<a+j$, then by Theorem 4.1, $u(a+j+2 \ell) \neq 0$ and $v(a+j+2 \ell) \neq 0$. Define $w(k)=v(a+j+2 \ell) u(k)-u(a+$ $j+2 \ell) v(k)$. Since $w(k)$ is a linear combination of $u(k)$ and $v(k)$, it is a solution of (1). However, $w(\gamma)=w(a+j)=w(a+j+\ell)=w(a+j+2 \ell)=0$ and so $w(k)$ must be the trivial solution of (1) by Theorem 4.1. Since $u(a+j+2 \ell)$ and $v(a+j+2 \ell)$ are nonzero, $u(k)$ and $v(k)$ must be constant multiples of each other.

Next, if $u(\gamma)=u(a+j)=u(\beta)=v(\gamma)=v(a+j)=v(\beta)=0$, where $\gamma<a+j<\beta-\ell$, then, by Corollary 4.5, $u(a+j+\ell) \neq 0$ and $v(a+j+\ell) \neq 0$. Define $w(k)=v(a+j+\ell) u(k)-u(a+j+\ell) v(k)$. Clearly, $w(\gamma)=w(a+j)=$ $w(a+j+\ell)=w(\beta)=0$, which contradicts Corollary 4.4 unless $w(k) \equiv 0$. But this means $u(k)$ and $v(k)$ are constant multiples of each other. This completes the proof.

\section{Recessive Solutions}

Let $u^{\left[\frac{m}{\ell}\right]}(k)$ be the solution of $(1)$ satisfying $u^{\left[\frac{m}{\ell}\right]}(m)=u^{\left[\frac{m}{\ell}\right]}(m+\ell)=u^{\left[\frac{m}{\ell}\right]}(m+$ $2 \ell)=0$ and $u^{\left[\frac{m}{\ell}\right]}(0)=\ell$ for $m \in[\ell, \infty)$. For each $m, u^{\left[\frac{m}{\ell}\right]}(k)$ exists and is unique. The existence is clear from Theorem 4.1 and a normalization, while the uniqueness follows from Theorem 4.7. Note that by construction

$$
0 \leq u^{\left[\frac{m}{\ell}\right]}(k) \leq 1 \text { for all } k \in[0, m+2 \ell] .
$$

Also, Theorem 4.1 implies that

$$
u^{\left[\frac{m}{\ell}\right]}(k) \geq u^{\left[\frac{m}{\ell}\right]}(k+\ell) \text { for all } k \in[0, \infty) .
$$

We now consider $m$ sequence $\left\{u^{\left[\frac{m}{\ell}\right]}(\ell)\right\}$. By $(37), 0 \leq u^{\left[\frac{m}{\ell}\right]}(\ell) \leq \ell$ for all $m \in[\ell, \infty)$, hence $\lim \sup \left\{u^{\left[\frac{m}{\ell}\right]}(\ell)\right\}$ exists, we call it $u(\ell)$. Then, there exists a subsequence $\left\{m_{1 t}\right\} \subseteq[\ell, \infty)$ such that $u^{\left[\frac{m_{1 t}}{\ell}\right]}(\ell) \rightarrow u(\ell)$ as $t \rightarrow \infty$. Next, consider the $m$-sequence $\left\{u^{\left[\frac{m}{\ell}\right]}(2 \ell)\right\}$. By (37), lim sup $\left\{u^{\left[\frac{m_{1 t}}{\ell}\right]}(2 \ell)\right\}$ exists, we call it $u(2 \ell)$. Also, there exists a subsequence $\left\{m_{2 t} \stackrel{t \rightarrow \infty}{\subseteq}\left\{m_{1 t}\right\}\right.$ such that 
$u^{\left[\frac{m_{2 t}}{\ell}\right]}(2 \ell) \rightarrow u(2 \ell)$ (and $\left.u^{\left[\frac{m_{2 t}}{\ell}\right]}(\ell) \rightarrow u(\ell)\right)$ as $t \rightarrow \infty$. In a similar fashion, by considering $\left\{u^{\left[\frac{m}{\ell}\right]}(3 \ell)\right\}$, we can arrive at a subsequence $\left\{m_{3 t}\right\}$ and a limit $u(3 \ell)$ such that $u^{\left[\frac{m_{3 t}}{\ell}\right]}(k) \rightarrow u(k)$ as $t \rightarrow \infty, k \in[\ell, 3 \ell]$. Clearly, $u^{\left[\frac{m_{3 t}}{\ell}\right]}(0)=\ell$ for all $t$.

Recall that by definition, for any $k$ and any $m$

$$
\begin{gathered}
u^{\left[\frac{m}{\ell}\right]}(k+2 \ell)-4 \alpha u^{\left[\frac{m}{\ell}\right]}(k+\ell)+6 \alpha^{2} u^{\left[\frac{m}{\ell}\right]}(k) \\
-4 \alpha^{3} u^{\left[\frac{m}{\ell}\right]}(k-\ell)+\alpha^{4} u^{\left[\frac{m}{\ell}\right]}(k-2 \ell)=\alpha^{2} p(k) u^{\left[\frac{m}{\ell}\right]}(k) .
\end{gathered}
$$

Consider (39) with $k=2 \ell$ and $m$ replaced by $m_{3 t}$. We can conclude that $\lim _{t \rightarrow \infty} u^{\left[\frac{m_{3 t}}{\ell}\right]}(4 \ell)$ exists, we call it $u(4 \ell)$. Now, replace $k$ by $3 \ell$ in (39) and conclude the existence of $\lim _{t \rightarrow \infty} u^{\left[\frac{m_{3 t}}{\ell}\right]}(5 \ell)=u(5 \ell)$. Proceeding inductively, we conclude that $\lim _{t \rightarrow \infty} u^{\left[\frac{m_{3 t}}{\ell}\right]}(k)=u(k)$ exists for any $k \in[0, \infty)$. Replacing $m$ by $m_{3 t}$ in (39) and letting $t \rightarrow \infty$, we conclude that $u(k)$ is a solution of (1). Also,

$$
u(k) \geq u(k+\ell) \geq 0 .
$$

This follows from (38) by replacing $m$ by $m_{3 t}$, fixing $k$ and letting $t \rightarrow \infty$. From (40), we conclude that

$$
\lim _{k \rightarrow \infty} u(k) \text { exists and we shall call it } L_{j} .
$$

In the following theorem we show that this $u(k)$ is a recessive solution of (1).

Theorem 5.1. The solution $u(k)$ constructed above is a recessive solution of (1). In addition $\Delta_{\alpha(\ell)} u(k), \Delta_{\alpha(\ell)}^{2} u(k)$ and $\Delta_{\alpha(\ell)}^{3} u(k)$ all monotonically approach zero as $k \rightarrow \infty$.

Proof. We will first show that (9) is satisfied. By (38) and Theorem 4.1, $u^{\left[\frac{m_{3 t}}{\ell}\right]}\left(m_{3 t}+3 \ell\right)<0$. Choosing $m_{3 t} \geq 3 \ell$ and using Lemma 3.5 with $a=m_{3 t}+\ell$, we can conclude that for any $k$ such that $2 \ell \leq k \leq m_{3 t}+\ell, \Delta_{\alpha(\ell)} u^{\left[\frac{m_{3 t}}{\ell}\right]}(k-\ell) \leq$ $0, \Delta_{\alpha(\ell)}^{2} u^{\left[\frac{m_{3 t}}{\ell}\right]}(k-\ell) \geq 0$ and $\Delta_{\alpha(\ell)}^{3} u^{\left[\frac{m_{3 t}}{\ell}\right]}(k-\ell) \leq 0$. Letting $t \rightarrow \infty$ implies that $u(k)$ satisfies (9) for $a=\ell$ and is recessive. We note that $u(k)$ also satisfies (9) for $a=0$. Concerning the monotonicity, we choose any $k \in[2 \ell, \infty)$ and any $m_{3 t} \geq k$. Then, $\Delta_{\alpha(\ell)}^{2} u^{\left[\frac{m_{3 t}}{\ell}\right]}(k-\ell) \geq 0$ which means $\Delta_{\alpha(\ell)} u^{\left[\frac{m_{3 t}}{\ell}\right]}(k) \geq$ $\alpha \Delta_{\alpha(\ell)} u^{\left[\frac{m_{3 t}}{\ell}\right]}(k-\ell)$ and hence $0 \leq-\Delta_{\alpha(\ell)} u^{\left[\frac{m_{3 t}}{\ell}\right]}(k) \leq-\alpha \Delta_{\alpha(\ell)} u^{\left[\frac{m_{3 t}}{\ell}\right]}(k-\ell)$. Taking the limit as $t \rightarrow \infty$ implies that $\Delta_{\alpha(\ell)} u(k)$ is monotonically decreasing in absolute value. By (41), since $u(k)$ monotonically approaches a finite 
limit, $\Delta_{\alpha(\ell)} u(k) \rightarrow 0$ as $k \rightarrow \infty$. The argument that $\Delta_{\alpha(\ell)}^{2} u(k)$ and $\Delta_{\alpha(\ell)}^{3} u(k)$ monotonically approach zero is similar.

Corollary 5.2. By Theorem 5.1 and Definition 2.5, this recessive solution $u(k)$ of (1) can be written as

$$
u(k-2 \ell)=L_{j}+\sum_{r=0}^{\infty}\left(\begin{array}{c}
r+3 \\
3
\end{array}\right) \frac{u(k+r \ell)}{\alpha^{r+4}} .
$$

Corollary 5.3. If $\sum^{\infty}(k+t \ell)^{3} p(k+t \ell)=\infty$, then, the recessive solution $u(k)$ of (1) constructed above approaches zero as $k \rightarrow \infty$.

Corollary 5.4. Suppose that $u(k)$ and $v(k)$ are two recessive solutions of (1) such that $u(a)=v(a)$. If $u(k) \geq v(k)$ for all $k \in[a, \infty)$, then $u(k) \equiv v(k)$.

Proof. Let $L_{j}=\lim _{k \rightarrow \infty} u(k)$ and $M_{j}=\lim _{k \rightarrow \infty} v(k)$. By hypothesis, $L \geq M$. Thus, if $w(k)=u(k)-v(k)$, then from (42) with $k=a+2 \ell$ we have $0 \geq L_{j}-M_{j}+\sum_{r=0}^{\infty}\left(\begin{array}{c}r+3 \\ 3\end{array}\right) \frac{u(a+2 \ell+r \ell)}{\alpha^{r+4}} \geq 0$. From this we conclude that $u(k) \equiv v(k)$.

\section{Acknowledgments}

Research Supported by National Board for Higher Mathematics, Department of Atomic Energy, Government of India, Mumbai.

\section{References}

[1] R.P Agarwal, Difference Equations and Inequalities, Marcel Dekker, New York, 2000.

[2] Jerzy Popenda and Blazej Szmanda, On the Oscillation of Solutions of Certain Difference Equations, Demonstratio Mathematica, XVII(1), (1984), $153-164$.

[3] Jerzy Popenda, Oscillation and Nonoscillation Theorems for Second-Order Difference Equations, Journal of Mathematical Analysis and Applications, 123(1), (1987), 34 - 38. 
[4] James Stewart, Single Variable Calculus: Concepts and Contexts, Brooks/Cole, USA, 2009.

[5] M.Maria Susai Manuel, G.Britto Antony Xavier and E.Thandapani, Theory of Generalized Difference Operator and Its Applications, Far East Journal of Mathematical Sciences, 20(2) (2006), 163 - 171.

[6] M.Maria Susai Manuel and G.Britto Antony Xavier, Recessive, Dominant and Spiral Behaviours of Solutions of Certain Class of Generalized Difference Equations, International Journal of Differential Equations and Applications, 10(4) (2007), 423-433.

[7] M.Maria Susai Manuel, G.Britto Antony Xavier and V.Chandrasekar, Generalized Difference Operator of the Second Kind and Its Application to Number Theory, International Journal of Pure and Applied Mathematics, 47(1) (2008), 127 - 140.

[8] M.Maria Susai Manuel, G.Britto Antony Xavier, D.S.Dilip and G.Dominic Babu, Oscillation, Nonoscillation and Growth of Solutions of Generalized Second Order Nonlinear $\alpha$-Difference Equations, Global Journal of Mathematical Sciences: Theory and Practical, 4(1), 2012, 211 - 225.

[9] M.Maria Susai Manuel, G.Britto Antony Xavier and D.S.Dilip, $\alpha$ Difference Operator And Its Application On Number Theory, J. of Mod. Meth. in Numer. Math., 3(2), (2012), 79 - 95.

[10] R.Pugalarasu, M.Maria Susai Manuel, V.Chandrasekar and G.Britto Antony Xavier, Theory of Generalized Difference operator of n-th kind and its applications in number theory (Part I), International Journal of Pure and Applied Mathematics, 64(1) (2010), 103 - 120.

[11] R.Pugalarasu, M.Maria Susai Manuel, V.Chandrasekar and G.Britto Antony Xavier, Theory of Generalized Difference operator of $n$-th king and its applications in number theory (Part II), International Journal of Pure and Applied Mathematics, 64(1) (2010), 121 - 132.

[12] Ronald E.Mickens, Difference Equations, Van Nostrand Reinhold Company, New York, 1990.

[13] Saber N.Elaydi, An Introduction To Difference Equations, Second Edition, Springer, 1999. 
[14] Walter G.Kelley, Allan C. Peterson, Difference Equations, An Introduction with Applications, Academic Press, inc 1991. 